\title{
Intelligent Monitoring and Predicting Output Power Losses of Solar Arrays Based on Particle Filtering
}

\author{
Hongzheng Fang, ${ }^{1,2,3}$ Bing Chen, ${ }^{4}$ Haodong Ma, ${ }^{2,3}$ and Liguo Zhang ${ }^{5}$ \\ ${ }^{1}$ School of Automation, Nanjing University of Science and Technology, Nanjing, China \\ ${ }^{2}$ Beijing Key Laboratory of High-Speed Transport Intelligent Diagnostic and Health Management, Beijing, China \\ ${ }^{3}$ Beijing Aerospace Measure \& Control Corp., Ltd., Beijing, China \\ ${ }^{4}$ College of Electronic Engineering, Naval University of Engineering, Wuhan, China \\ ${ }^{5}$ School of Electronic Information and Control Engineering, Beijing University of Technology, Beijing, China
}

Correspondence should be addressed to Liguo Zhang; zhangliguo@bjut.edu.cn

Received 10 April 2013; Revised 2 June 2013; Accepted 4 June 2013

Academic Editor: Chengjin Zhang

Copyright (C) 2013 Hongzheng Fang et al. This is an open access article distributed under the Creative Commons Attribution License, which permits unrestricted use, distribution, and reproduction in any medium, provided the original work is properly cited.

\begin{abstract}
Solar arrays are the main source of energy to the on-orbit satellite, whose output power largely determines the life cycle of onorbit satellites. Monitoring and further forecasting the output power of solar arrays by using the real-time observational data are very important for the study of satellite design and on-orbit satellite control. In this paper, we firstly describe the dynamical model of output power with summarizing the influencing factors of attenuation for solar arrays and elaborating the evolution trend of influencing factors which change with time. Based on the empirical model, a particle filtering algorithm is formulated to predict the output power of solar arrays and update the model parameters, simultaneously. Finally, using eight-year observational data of voltage and current from a synchronous on-orbit satellite, an experiment is carried out to illustrate the reliability and accuracy of the particle filtering method. Comparative results with classical curve fitting also are presented with statistical root mean square error and mean relative error analysis.
\end{abstract}

\section{Introduction}

Solar arrays (SA) are the main source of energy to the on-orbit satellites, whose output power determines the life cycle of satellites in use to a great extent. In the whole life cycle of satellite, the power of satellite load's demand does not change significantly, while the SA output power decays constantly. At the initial and middle stages of satellite life, the power demand of satellite load is less than the SA output power, which can make sure of the power balance of on-orbit satellite. However, when the satellite is in the end stage of life, the attenuation of SA output power is obvious which may affect the normal operation of the satellite $[1,2]$. Therefore, it is a very important work in the areas of satellite design and on-orbit satellite control to study the attenuation model of output power, to dynamically update the model based on the real-time observational data of power, and further to predict the output power.
Prediction models about the attenuation of SA output power can be divided into three categories, which are the data-driven prediction method, prediction method based on model, and dynamic prediction method based on model and data. Among them, data-driven prediction methods, such as the Autoregressive Moving Average model (ARIMA), neural network, and other methods of pattern recognition, have been proved applicative to both diagnostics and prognostics in many fields $[3,4]$, but those methods make it hard to establish a general empirical model about SA output power varying with time. Main tasks of prediction methods based on model are to analyze the influencing factors of SA output power attenuation and their effect on power and further to establish an empirical model about SA output power varying with time, through a lot of simulation and historical measurement data. Then according to data from a specific system and using curve fitting to estimate the parameters of the empirical model, the concrete formula of SA output 
power varying with time can be obtained, by which the output power at any time point can be achieved $[2,5]$. This method actually belongs to static modeling. However, with the SA running in the complicated space environment and subjecting to a number of uncertain factors, such as particle radiation and ultraviolet irradiation, it is difficult to meet the forecast accuracy requirement with the static model. So that satellite life could not be accurately predicted by prediction methods based on model. On the basis of the empirical model, dynamic prediction methods, such as the Extended Kalman Filter (EKF) $[6,7]$ and particle filter (PF) $[8,9]$, can dynamically update model parameters and predict output power simultaneously using historical data. And PF could be a solution to the nonlinear and dynamic model with nonGaussian noise. Therefore, to the nonlinear and dynamic SA output power model with non-Gaussian noise, the method based on PF will be a perfect selection.

This paper summarizes the influencing factors of SA power attenuation, elaborates the factors' trend with time and their effect on output power, and further describes the empirical model about output power varying with time. Next, it puts forward the algorithm of PF dynamic prediction based on the empirical model and historical data. By using the algorithm, model parameter updating and output power realtime prediction can be carried out. Finally, using eight years data of SA voltage and current from a synchronous satellite in orbit, the validity of empirical model about the attenuation of SA output power varying with time was verified based on curve fitting. In addition, the reliability and accuracy of the dynamic prediction method based on PF in model parameters updating and output power real-time prediction were also proved out.

\section{Empirical Model}

SA output power is changing along with three impact factors, which are the solar sunlight intensity, solar incident angle, and attenuation factor [2]. The first two parts can be got through the orbit calculation and have a changing trend with annual cycle, which makes the SA output power change with a cycle of one year as well. Attenuation factor is the comprehensive of particle radiation, ultraviolet irradiation, micrometeoroid collision, and alternating between cold and hot, and so on [10]. It can be captured through a series of simulations about SA on the ground, like particle radiation experiment or through analyzing remote sensing data from on-orbit satellite [4]. Based on the method of analyzing onorbit data to estimate the empirical model, the empirical model of SA output power $[2,5,11]$ can be described as follows:

$$
P=V I=P_{0} J \cos (\theta) \eta
$$

where $V$ and $I$ denote comprehensive SA voltage and current in orbit. $P$ and $P_{0}$ show output power and the maximum output power of SA on-orbit. $J, \theta$, and $\eta$ denote the solar sunlight intensity coefficient, solar incident angle, and attenuation factor, respectively.
Since the space environment is very complex, the environmental impact on SA is shown in Table 1.

The earth runs along an elliptic orbit with the sun as the center and a cycle of one year, so that the solar sunlight intensity coefficient $J$ changes with annual cycle as well, as shown in formula (2). The solar incident angle $\theta$ is the included angle between the vector of sunlight incident and the SA normal vector. And because of the fact that the earth executes revolution and rotation at the same time, $\theta$ changes between $-23.44^{\circ}$ and $23.44^{\circ}$ with annual cycle, as shown in formula (3).

$$
\begin{gathered}
J(t)=\frac{1354+45 \cos \left(\left(t+T_{0}\right)\left(360^{\circ} / 365.25\right)\right)}{1353}, \\
\theta(t)=\arcsin \left(\sin \left(23.44^{\circ}\right) \sin \left[\left(t+T_{0}+101\right) \frac{360^{\circ}}{365.25}\right]\right),
\end{gathered}
$$

where $t$ denotes the number of days of SA in orbit and $T_{0}$ shows the day difference between the date of spacecraft first into space and January 3 in the same year.

Set the initial time that is the date of spacecraft first into space as January 1 in a year, namely, $T_{0}=2$. The trend of $J$ and $\theta$ varying with the days of SA on-orbit, from January 1 in the first year to December 31 in the eighth year, is shown in Figures 1 and 2, respectively. If not specified, the unit of abscissa of each graphic shown in this paper is "days of SA on-orbit $t$."

The exponential function concerning days of SA on-orbit $t$ can represent the variation of $\eta$, as shown in the following:

$$
\eta(t, \xi, \sigma)=\xi+(1-\xi) e^{-t / \sigma}
$$

where $\xi$ and $\sigma$ are the parameters to donate the trend of attenuation factor index change. Supposing that from January 1 in the first year to December 31 in the eighth year, parameters of $\xi$ and $\sigma$ remain unchanged. Set $\xi=0.696$ and $\sigma=1000$, and the trend of $\eta$ varying with $t$ is shown in Figure 3.

According to formulas from (1) to (4), the empirical model about $P$ varying with time $t$, which is exponential decay along with $t$ and periodic variation with a cycle of one year, can be obtained. Therefore, at any time point of $t$, the empirical model about $P$ only exists 2 unknown parameters. If the two parameters can be accurately estimated through analysis of on-orbit historical data, it can predict $P$ in the next point of time accurately.

Based on the simulation shown from Figures 1 to 3 , the trend of SA output power $P$ varying along with $t$ can be shown in Figure 4 from January 1 in the first year to December 31 in the eighth year, when setting $P_{0}$ as $1100(\mathrm{~W})$.

\section{Dynamic Prediction Based on PF}

SA output power model is a nonlinear and dynamic model with non-Gaussian noise. In Section 2, it has been indicated that attenuation factor is the comprehension of many uncertain factors. In the ideal state, $\xi$ and $\sigma$ in the function of 
TABLE 1: Impact factors on SA output power.

\begin{tabular}{|c|c|c|c|}
\hline Impact factors & Main relevant influencing factors & $\begin{array}{l}\text { The trend varying with the days } \\
\text { of SA on-orbit }\end{array}$ & The relation to the output power \\
\hline $\begin{array}{l}\text { Solar sunlight intensity } \\
\text { coefficient }\end{array}$ & $\begin{array}{l}\text { The direction of the sun } \\
\text { Distance between the Earth and } \\
\text { the Sun }\end{array}$ & $\begin{array}{l}\text { Trigonometric variation with a } \\
\text { cycle of one year }\end{array}$ & $\begin{array}{l}\text { Impact factor determining the } \\
\text { periodic change with a cycle of } \\
\text { one year }\end{array}$ \\
\hline Solar incident angle & Solar incident angle & & \\
\hline Attenuation factor & $\begin{array}{l}\text { Particle radiation } \\
\text { Ultraviolet irradiation } \\
\text { Micro meteoroid collision } \\
\text { Alternating between cold and hot } \\
\text { Space pollution to SA }\end{array}$ & Exponential decay & $\begin{array}{l}\text { Impact factor determining the } \\
\text { attenuation along with the } \\
\text { number of days of SA in orbit }\end{array}$ \\
\hline
\end{tabular}

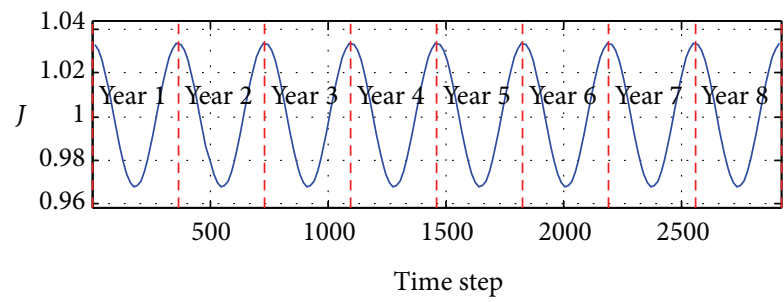

Figure 1: The trend of solar sunlight intensity coefficient $J$.

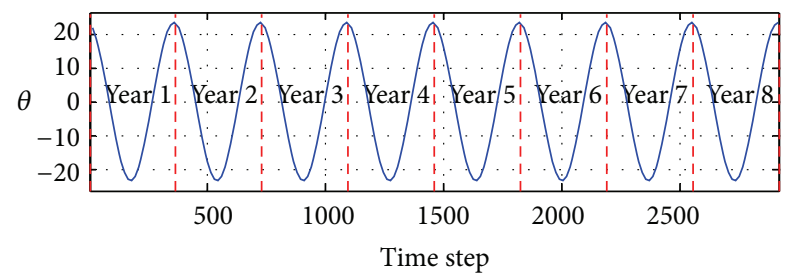

FIgURE 2: The trend of solar incident angle $\theta$.

attenuation factor varying with days of SA on-orbit remain unchanged; namely, that attenuation factor is determined only by days of SA on-orbit. Obviously, it goes against the practical situation that the space environment is very complicated and the environmental impact on SA is comprehensive, so that it cannot reflect the dynamic trend of SA output power.

The PF method constantly adjusts the weight and position of particles according to the real-time observed data and then modifies the conditional distribution of particles to estimate the related parameters and the state of system. This algorithm is one of recursive filtering algorithms, which can be used to estimate the state and parameters regarding arbitrary nonlinear and non-Gaussian random system. Therefore, it can solve the problem of SA output power model updating and output power prediction perfectly. The dynamic prediction algorithm of SA output power based on $\mathrm{PF}[8,9]$ is introduced below.

PF regards both the unknown parameters of the empirical model and the real-time prediction results of output power as the PF state equations and treats the real-time observation of output power as the observation equation. First of all, the state of particles update can be denoted in formula (5) and

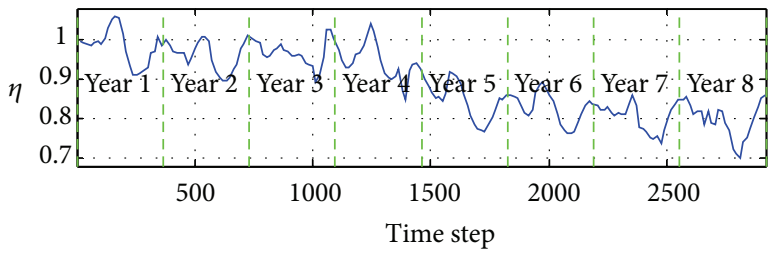

FIGURE 3: The trend of attenuation factor $\eta$.

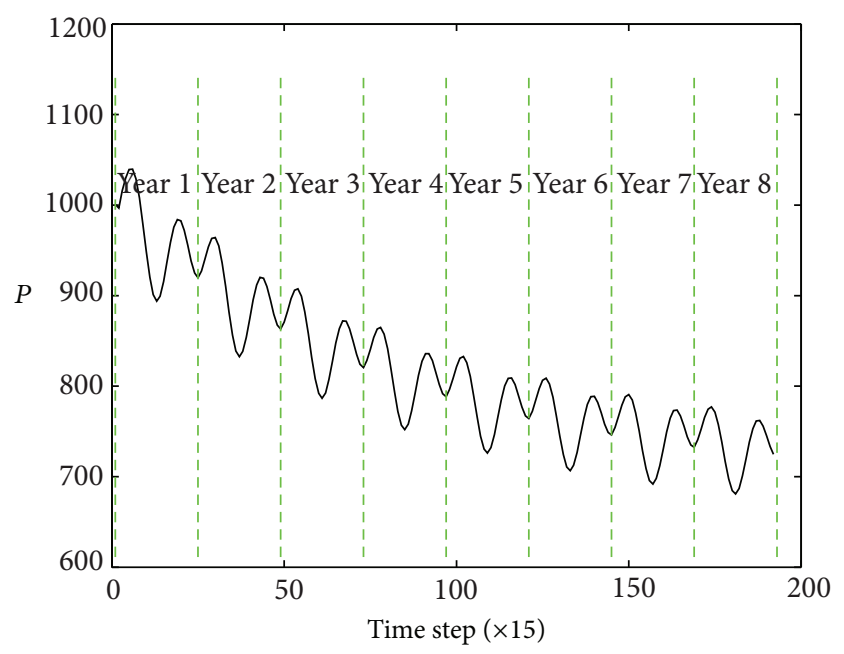

FIGURE 4: The trend of SA output power $P$ (unit of ordinate: W).

(6). Next, on the premise that the probability distribution of the observation noise and the results of particle prediction are approximate, the probability distribution function of observation noise is regarded as that of particle prediction results, in order to estimate the weight of particles, as shown in formula (7). Thirdly, particles are reselected, to particles with a bigger weight, and more sampling will be executed, while little or no sampling will be done to particles with smaller weight. Then according to formula (8), the model parameters can be updated and the output power can be predicted. Repeat operations mentioned above, to implement the correction of model parameters and output power prediction in the next moment, as shown in Figure 5. 


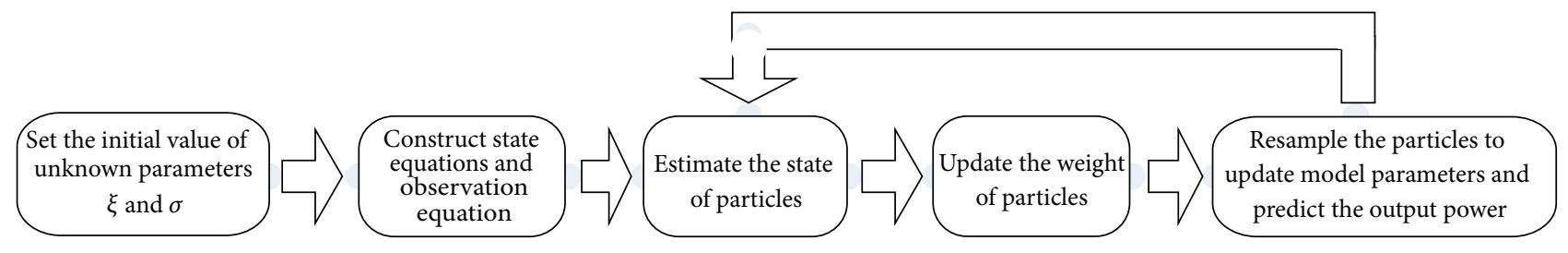

FIGURE 5: The process of model parameters updating and output power prediction based on PF.

Set the initial value of unknown parameters $\xi$ and $\sigma$ in the empirical model as $\xi_{0}$ and $\sigma_{0}$, respectively. $\xi_{i+1}$ and $\sigma_{i+1}$ represent the updating values of model parameters at the time point $i$. Both process noise and observation noise obey normal distribution, and they are described as $n_{p} \sim$ $N\left(0, s_{p}\right)$ and $n_{m} \sim N\left(0, s_{m}\right)$, and the number of particles is set as $l$. At the time point $i$, the actual measured value, the predicted value, and derivative of the output power are denoted as $P_{i}, \widetilde{P}_{i+1}, d P_{i} / d t$, respectively. The related formulas of $\mathrm{PF}$ algorithm show are below.

According to the conservation relationship, we can define the state equations as follows:

$$
\begin{gathered}
\xi(i+1, j)=\xi(i, j)+n_{p}, \quad \xi(0, j)=\xi_{0}+n_{p}, \\
\sigma(i+1, j)=\sigma(i, j)+n_{p}, \quad \sigma(0, j)=\sigma_{0}+n_{p}, \\
\widetilde{P}_{i+1}(i+1, j)=P_{i}+\frac{d P_{i}}{d t} \Delta t+n_{p}, \\
i=0,1,2, \ldots ; j=1,2, \ldots l
\end{gathered}
$$

and the output power is defined as the observation variable,

$$
P_{i+1}=P_{i+1}+n_{m}, \quad i=0,1,2, \ldots
$$

Through the output power $\widetilde{P}$, we update the weight of particles as follows:

$$
\begin{aligned}
& w(i+1, j)=\frac{\bar{w}(i+1, j)}{\sum_{j=1}^{l} \bar{w}(i+1, j)}, \quad i=0,1,2, \ldots ; j=1,2, \ldots l, \\
& \bar{w}(i+1, j)=\frac{1}{\sqrt{2 \pi} s_{m}} e^{-\left(\widetilde{P}_{i+1}(i+1, j)-P_{i+1}\right)^{2} / 2 s_{m}{ }^{2}} .
\end{aligned}
$$

By the weight of particles, we can update the model parameters and predict the output power,

$$
\begin{gathered}
\xi_{i+1}=\frac{\sum_{j=1}^{l} c_{j} \xi_{i+1}(i+1, j)}{l}, \quad \sigma_{i+1}=\frac{\sum_{j=1}^{l} c_{j} \sigma_{i+1}(i+1, j)}{l}, \\
\widetilde{P}_{i+1}=\frac{\sum_{j=1}^{l} c_{j} \widetilde{P}_{i+1}(i+1, j)}{l}, \quad 0 \leq c_{j} \leq l \\
\sum_{j=1}^{l} c_{j}=1, \quad i=0,1,2, \ldots ; j=1,2, \ldots l .
\end{gathered}
$$

If the label of parameters or the output power is $(i, j)$, it denotes the value of corresponding particle whose label is $j$ at the time point $i . c_{j}$ shows times that the particle whose label $j$ is reselected. $\Delta t$ is a constant, $\Delta t=t_{i+1}-t_{i}$, and $t_{i}$ denotes days of SA on-orbit at time point $i$. In (8), the parameter $c$ is the frequency of the particle, and the particle which is selected more frequently has the more weight: namely, the parameter $w$ is bigger.

\section{Experimental Results}

We calculated SA output power by using 8-year data of SA onorbit voltage and current data from January 1 in the 1st year to December 31 in the 8 th year stemming from a synchronous on-orbit satellite. Then, considering the minimum unit of time as half a month, we calculated and captured the mean sequence of output power. Based on the mean sequence of output power obtained above from 8 years of SA on-orbit, we combined the empirical model, as described in Section 2, with dynamic prediction method based on PF to update parameters of the model and predicted the real-time output power. And through several times of simulations, the method is proven to be reliable and accurate in output power forecast.

4.1. The Description of Simulation Data. We used formula (1) $P=V I$ to calculate SA output power, and the results are shown in Figure 6, and we set the minimum unit of time as half a month, and then we calculated and captured the mean sequence of output power using formula (1) $P=V I$, as shown in Figure 7.

4.2. Modeling and Analysis. Based on the mean sequence of SA output power described before, we used the method of curve fitting to estimate the value of $\xi$ and $\sigma$. With the root mean square error (RMSE) and the mean relative error (MRE) of attenuation factor fitting as the standards, we verified the validity of the empirical model about the attenuation of SA output power. Then, we adopted the proposed dynamic prediction method based on PF to update model parameters and predicted the real-time output power. Through several times of simulations and summarizing the statistical data of RMSE and MRE on output power real-time prediction, the method is proven to be reliable and accurate in output power forecast.

4.2.1. Estimation Model Parameters Based on Curve Fitting. We can get the actual value of attenuation factor by normalizing the SA output power by the solar sunlight intensity and solar incident angle, as shown in formula (9). Next, in accordance with formula (4), we can estimate the value of $\xi$ 


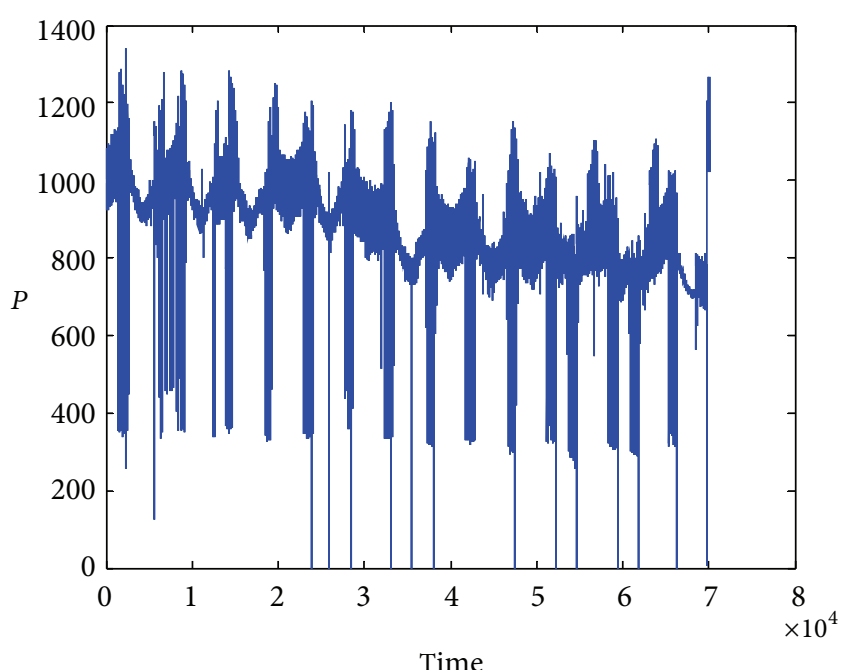

Figure 6: The eight years of original SA output power (unit of abscissa: hour; unit of ordinate: W).

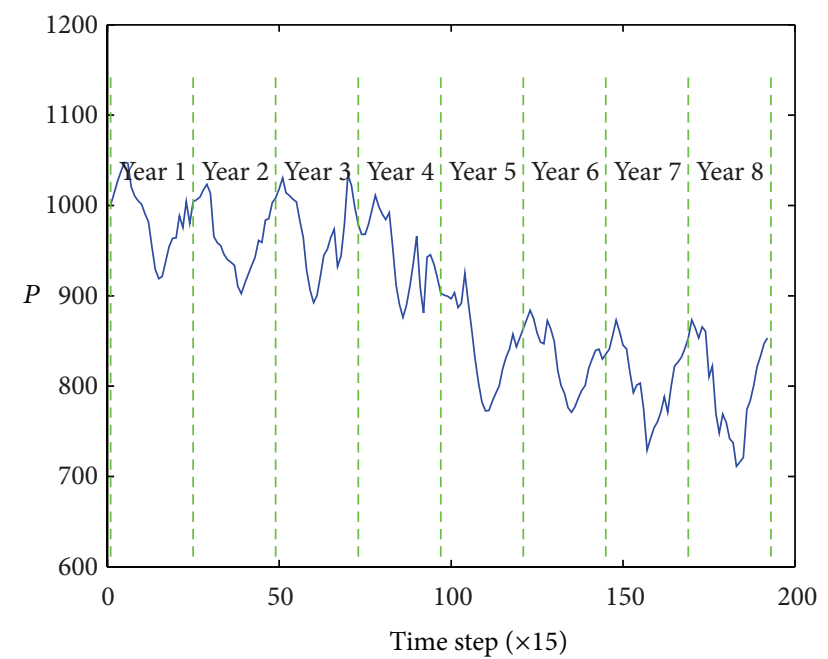

FIGURE 7: The mean sequence of SA output power (unit of ordinate: W).

and $\sigma$ using curve fitting and get the optimal results $\xi_{0}$ and $\sigma_{0}, \xi_{0}=0.696, \sigma_{0}=1278.375$, as shown in Figure 8:

$$
\eta(t, \xi, \sigma)=\frac{P(t)}{P_{0} \cdot J(t) \cos (\theta(t))} .
$$

Among them, $P(t)$ denote the actual value of output power in time point $t$ of SA on-orbit.

The error RMSE and MRE were 0.09064 and 0.095909, respectively, so that the fitting effect is good; that is to say that the attenuation factor changes with a tendency of exponential attenuation over SA on-orbit time $t$, indicating that the empirical model described in Section 2 shows the trend of output power attenuation of SA on-orbit perfectly.

4.2.2. Dynamic Prediction Based on PF and the Results Analysis. By using the proposed method described in Section 3, we

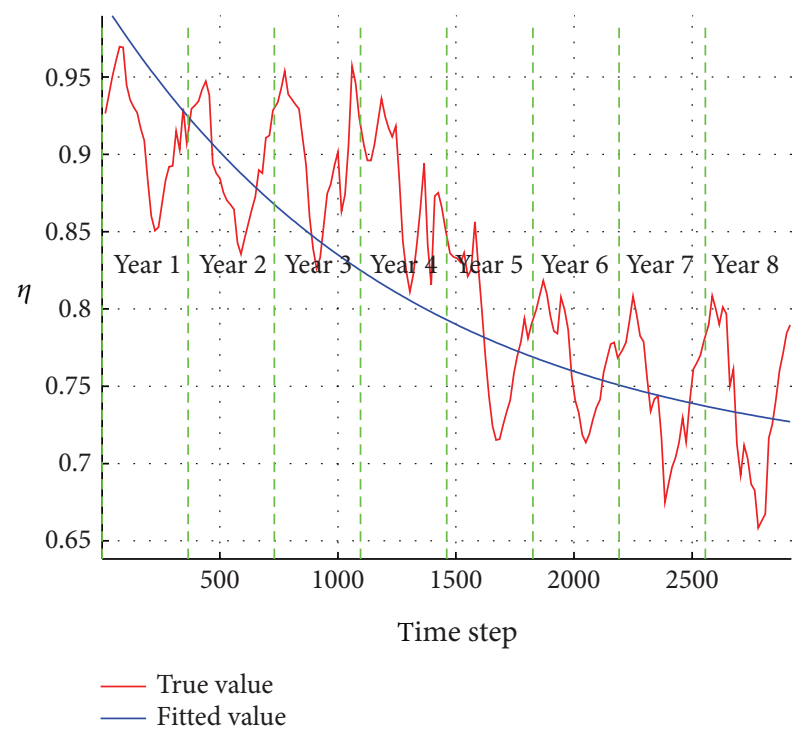

Figure 8: Contrast diagram of the actual fitted value about $\eta$.

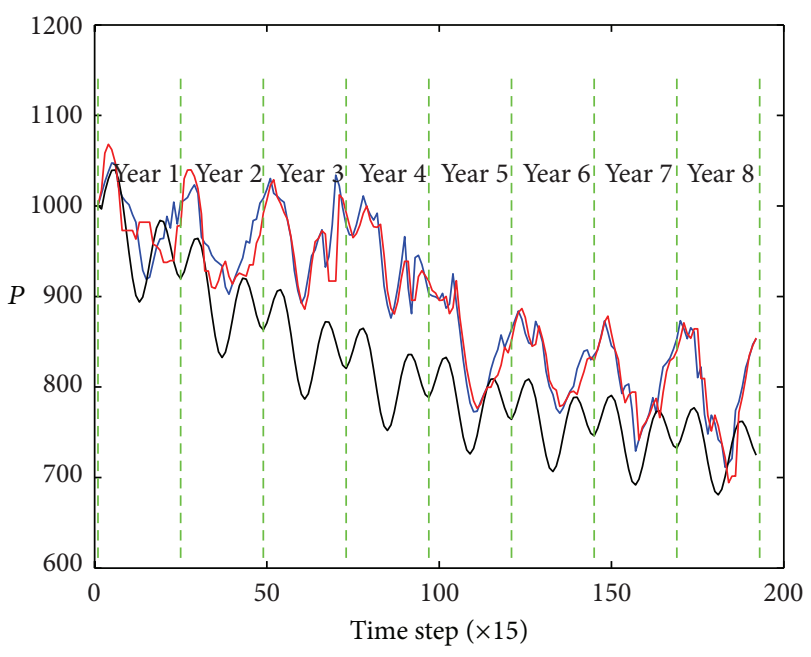

True value
_ Estimated value based on empirical model
Estimated value based on PF dynamic model

FIGURE 9: Real-time prediction results (Unit of ordinate: W).

predicted the output power and updated model parameters in time. Prediction results of SA output power and attenuation factor are shown in Figures 9 and 12, respectively. And the real-time renewal processes of model parameters $\xi$ and $\sigma$ were shown in Figures 10 and 11, in which 2 methods, named after fixed empirical model and PF dynamic model respectively, were shown. To the fixed empirical model, the model parameters $\xi$ and $\sigma$ are all fixed and equal to the optimal value $\xi_{0}$ and $\sigma_{0}$ mentioned before, as shown in Figures 10 and 11 with red “*”. In contrast with the actual value and the results of fixed empirical model, it is known that through dynamic correction of empirical model according to historical data acquired in time, this proposed method ideally 


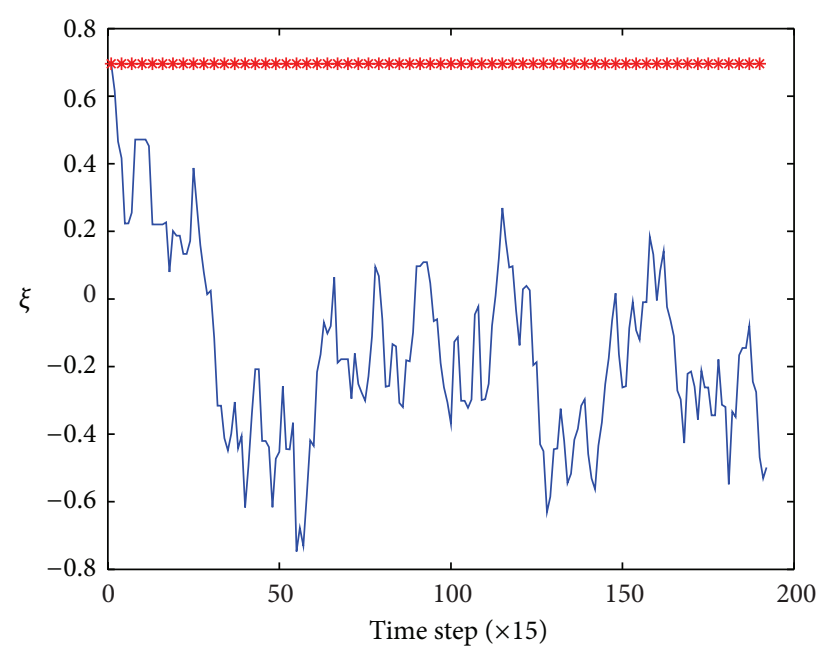

_ Estimated value based on PF dynamic model

* Estimated value based on empirical model

FIGURE 10: Estimated value of $\xi$.

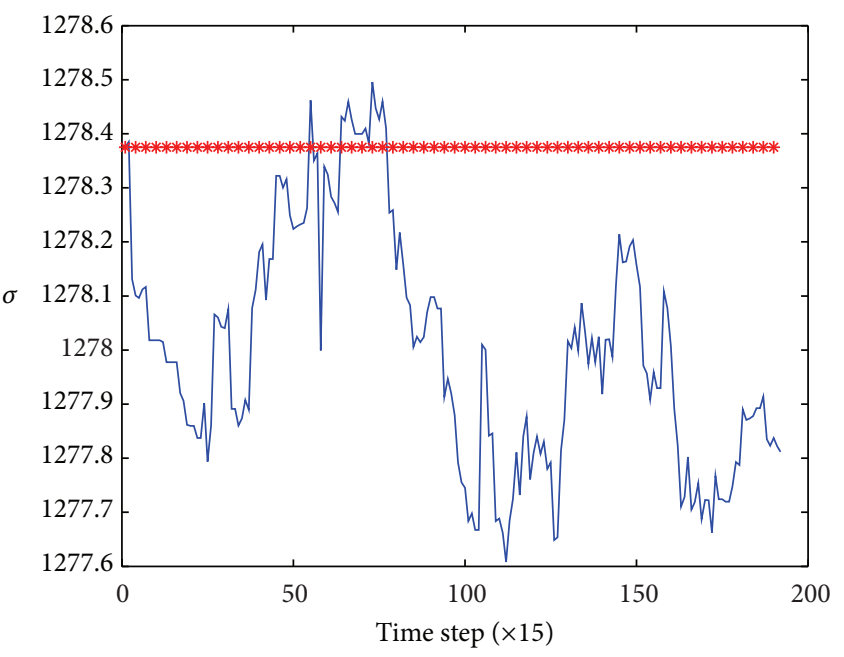

_ Estimated value based on PF dynamic model

* Estimated value based on empirical model

Figure 11: Estimated value of $\sigma$.

forecasts the output power and attenuation factor, as shown in Figures 9 and 12.

The statistical data of RMSE and MRE on output power real-time prediction is summarized by several times of simulations based on dynamic prediction PF method, and the results are that the mean of RMSE and MRE were 23.9516 and 0.0189 , respectively, and that the fluctuation variance of RMSE and MRE were 0.5551 and 0.000502, respectively. Therefore, the output prediction method is not only accurate but also has high stability; namely, this method has both good reliability and accuracy in output prediction. The prediction error of dynamic prediction model based on PF and fixed empirical model was shown in Table 2. Consequently, the dynamic prediction model based on PF proposed in this
TABLE 2: Prediction error.

\begin{tabular}{lcc}
\hline Prediction method & RMSE & MRE \\
\hline Fixed empirical model & 86.8747 & 0.082066 \\
PF dynamic model & $\approx 23.9516$ & $\approx 0.0189$ \\
\hline
\end{tabular}

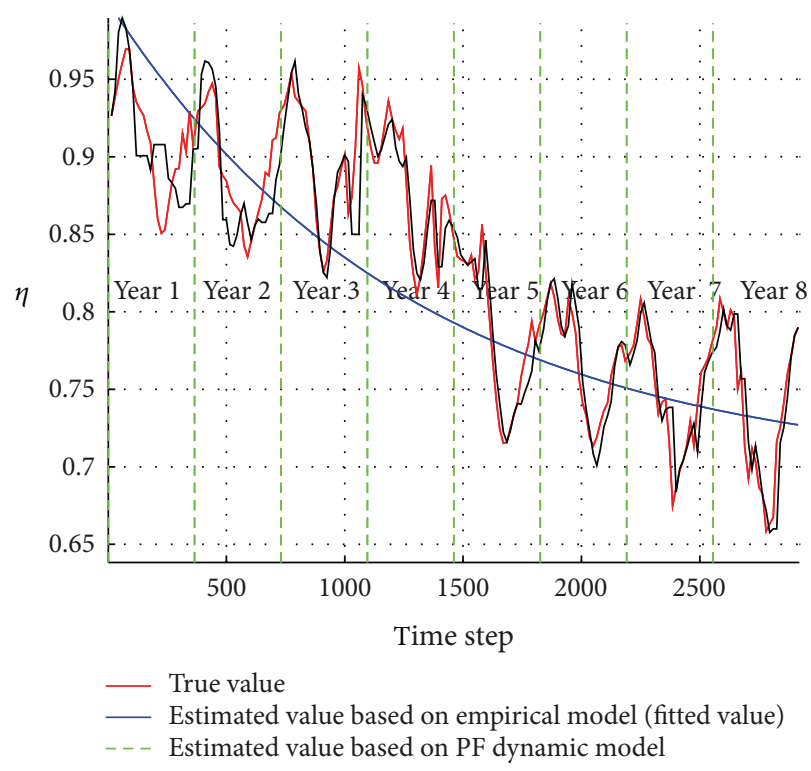

FIGURE 12: Estimated value of attenuation factor $\eta$.

paper is more suitable to describe the trend of output power and the attenuation factor varying with time.

\section{Conclusion}

This paper summarizes the influencing factors concerning the attenuation of solar arrays output power, elaborates the factors' trend with time and their effect on power, and further describes the empirical model about output power varying with time. Besides, it puts forward the algorithm of particle filter dynamic prediction based on the empirical model and historical data. By using the algorithm, model parameter updating and output power real-time prediction can be carried out. Finally, using eight years data of solar arrays' voltage and current from a synchronous satellite in orbit, the validity of empirical model about the attenuation of solar arrays output power varying with time was verified based on curve fitting. Then the proposed dynamic prediction method based on particle filter is used to update model parameters and predict the real-time output power. Through several times of simulations, it indicates that through dynamic correction of empirical model according to historical data acquired in time, this method can ideally forecast the output power and attenuation factor, proving out the reliability and accuracy of particle filter dynamic model in model parameters updating and output power real-time prediction. It is of important reference value in the areas of satellite energy measurement prediction. The results can make a contribution to the satellite design and on-orbit satellite control. 


\section{References}

[1] V. A. Shuvalov, G. S. Kochubei, V. V. Gubin, and N. A. Tokmak, "Power losses of solar arrays under the action of an environment in a geosynchronous Orbit," Cosmic Research, vol. 43, no. 4, pp. 259-267, 2005.

[2] V. A. Letin, "Optical, radiation and thermal cycling losses of power Si solar array returned from orbital station "Mir" after 10. 5 years of operation," Solar Engineering, no. 2, pp. 3-12, 2004.

[3] J. D. Kozlowski, "Electrochemical cell prognostics using online impedance measurements and model-based data fusion techniques," in Proceedings of IEEE Aerospace Conference, vol. 7, pp. 3257-3270, 2003.

[4] H. Fang, Y. Xiong, K. Luo, and L. Han, "Study of the long-term performance prediction methods using the spacecraft telemetry data," in Proceedings of the IEEE Prognostics and System Health Management Conference (PHM '12), 2012.

[5] M. H. Jphnson and J. K. Ball, "Combined release and radiation effects satellite (CRRES)," Speacecraft and Rockets, vol. 29, no. 4, pp. 556-563, 1992.

[6] B. S. Bhangu, P. Bentley, D. A. Stone, and C. M. Bingham, "Nonlinear observers for predicting state-of-charge and stateof-health of lead-acid batteries for hybrid-electric vehicles," IEEE Transactions on Vehicular Technology, vol. 54, no. 3, pp. 783-794, 2005.

[7] G. L. Plett, "Extended Kalman filtering for battery management systems of LiPB-based HEV battery packs-part 1. Background," Journal of Power Sources, vol. 134, no. 2, pp. 252-261, 2004.

[8] B. Saha and K. Goebel, "Modeling Li-ion battery capacity depletion in a particle filtering framework," in Proceedings of Annual Conference of the Prognostics and Health Management Society, 2009.

[9] B. Saha, K. Goebel, S. Poll, and J. Christophersen, "Prognostics methods for battery health monitoring using a Bayesian framework," IEEE Transactions on Instrumentation and Measurement, vol. 58, no. 2, pp. 291-296, 2009.

[10] P. Mei, W. Weiwei, W. Jing, and L. Ming, "Study on attenuation factor of Si solar array for satellite in sun synchronous orbit," Spacecraft Engineering, vol. 20, no. 5, pp. 61-67, 2011.

[11] B. Peter, The Geostationary Applications Satellite, Cambridge University Press, Cambridge, UK, 1988. 


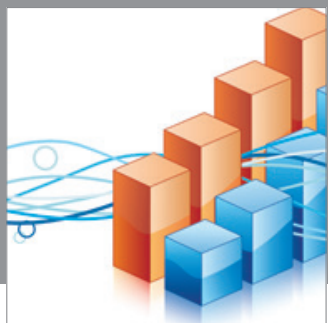

Advances in

Operations Research

mansans

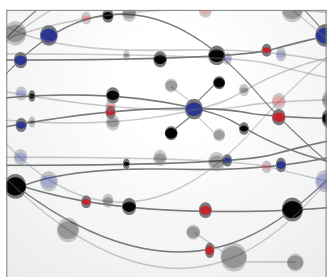

The Scientific World Journal
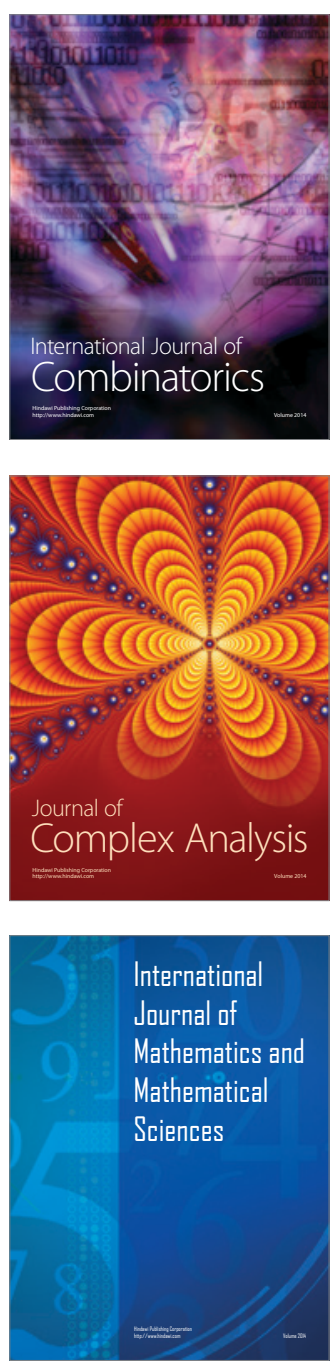
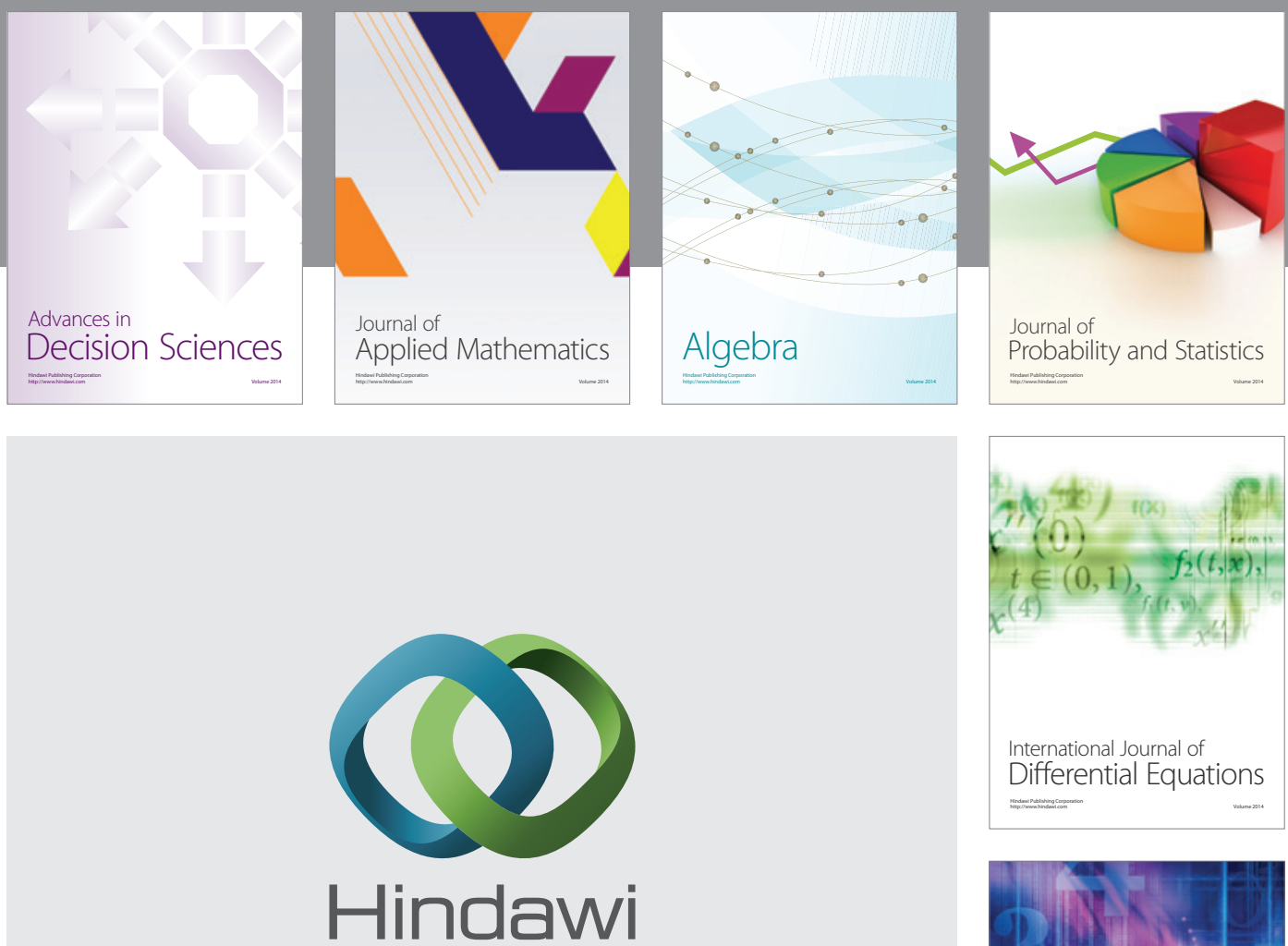

Submit your manuscripts at http://www.hindawi.com
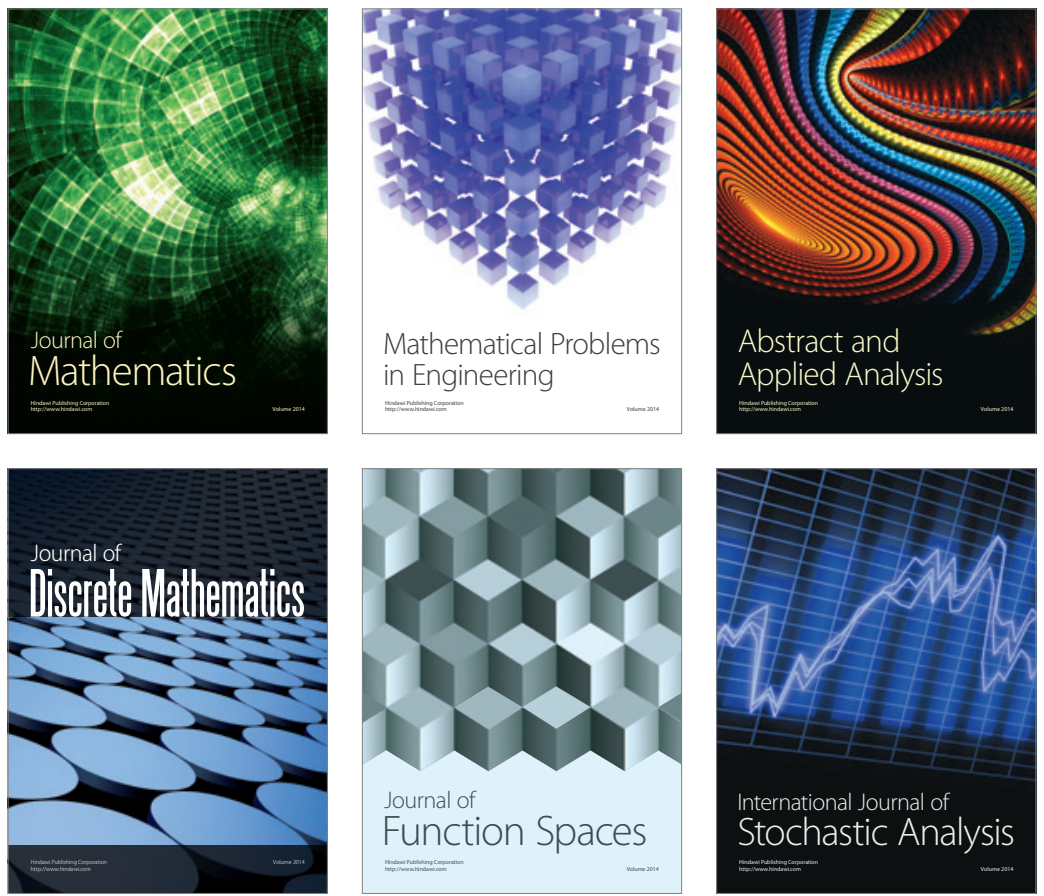

Journal of

Function Spaces

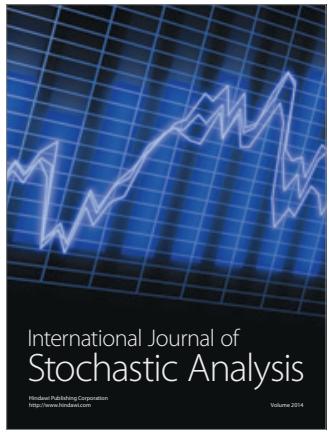

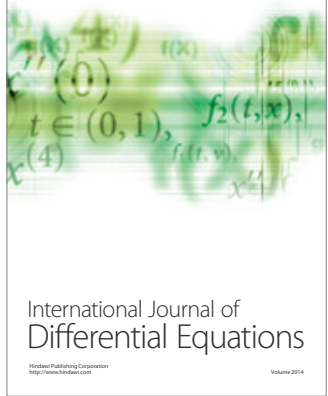
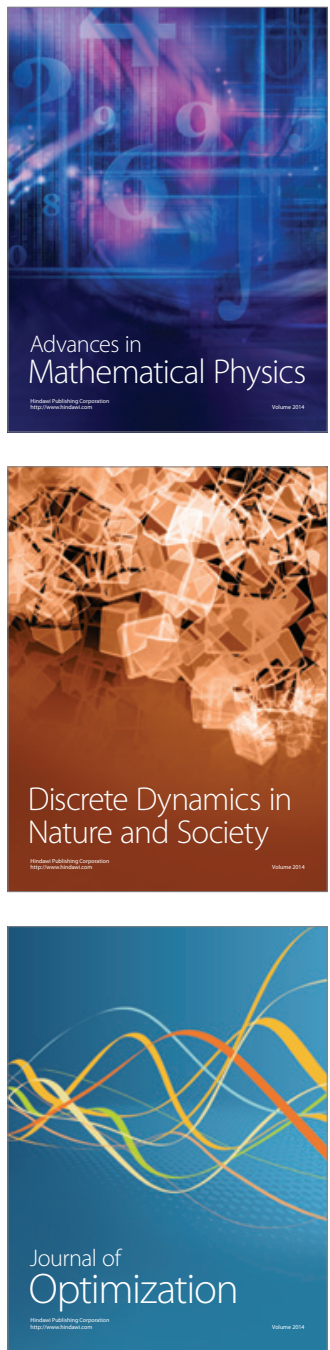